\title{
Comparação das estimativas de atividade física e comportamento sedentário em adultos brasileiros no VIGITEL e PNS, Brasil, 2013
}

\author{
Comparison of physical activity and sedentary behavior estimates in Brazilian adults \\ from VIGITEL and PNS, Brazil, 2013
}

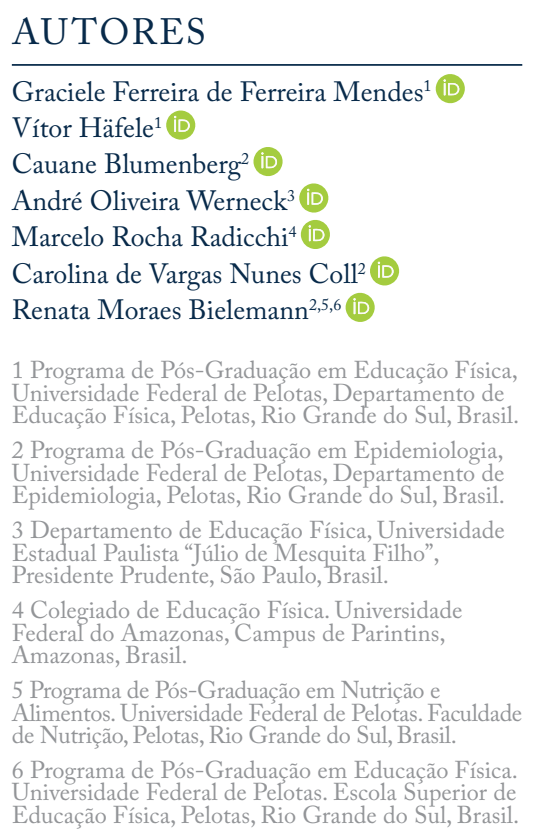

\section{CONTATO}

Graciele Ferreira Mendes

graciferreiramendes@hotmail.com

Programa de Pós-Graduação em Educação

Física, Universidade Federal de Pelotas.

Rua Luís de Camões, 625, Pelotas, Rio

Grande do Sul, Brasil. CEP: 96055-630.

DOI

$10.12820 /$ rbafs.23e0062

\begin{abstract}
RESUMO
O objetivo do presente estudo foi comparar as estimativas de atividade física e comportamento sedentário obtidas pelos inquéritos nacionais VIGITEL e PNS. Foram utilizados dados do VIGITEL 2013 e PNS 2013, conduzidos entre adultos ( $\geq 18$ anos). Os desfechos foram: atividade física no lazer, deslocamento ativo para o trabalho, inatividade física no lazer e tempo de televisão. Sexo, idade, escolaridade e índice de massa corporal foram as variáveis independentes. Para a estatística, utilizou-se proporções (IC95\%) e Coeficiente de Correlação de Concordância de Lin (CCC). As estimativas de deslocamento ativo para o trabalho e de tempo de TV apresentaram concordância moderada entre os inquéritos ( $\mathrm{CCC}=0,515 ; \mathrm{CCC}=0,478$, respectivamente), enquanto as estimativas de atividade física no lazer e inatividade no lazer apresentaram concordância baixa $(\mathrm{CCC}=0,060 ; \mathrm{CCC}=0,054$, respectivamente). As estimativas obtidas entre os inquéritos PNS e VIGITEL sobre desfechos relacionados à atividade física e comportamento sedentário foram de concordância moderada a baixa, não sendo os resultados obtidos comparáveis.
\end{abstract}

Palavras-chave: Exercício; Estilo de vida sedentário; Adulto; Estilo de vida saudável; Inquéritos epidemiológicos.

\begin{abstract}
The aim of the present study was to compare estimates of physical activity and sedentary behavior by two Brazilian surveys, VIGITEL PNS. Were used data from VIGITEL 2013 and PNS 2013, conducted among adults $(\geq 18 y)$. Were adopted as outcomes: leisure-time physical activity, active transportation to work, leisure-time physical inactivity and TV time. Sex, chronological age, race, educational status, marital status and body mass index were independent variables. For statistical analyses, proportions (IC95\%) and Lin's concordance correlation coefficient $(C C C)$ were calculated. The estimates of active transportation for work and $T V$ viewing showed moderate agreement between the surveys $(C C C=0.515 ; C C C=0.478$, respectively), while estimates of leisure activity and leisure physical inactivity showed a low agreement $(C C C=0.060 ; C C C=0.054$, respectively). Agreement between the estimates of physical activity and sedentary behavior provided by the VIGITEL and PNS ranged from moderate to poor and the results are not comparable.
\end{abstract}

Keywords: Exercise; Sedentary lifestyle; Adult; Healthy lifestyle, Health surveys.

\section{(cc) BY-NC-SA}

Este obra está licenciado com uma Licença

Creative Commons Atribuição-NãoComercial-

CompartilhaIgual 4.0 Internacional.

\section{Introdução}

Níveis insuficientes de atividade física são associados a diversos desfechos de risco a saúde, sendo que no
Brasil, se a inatividade física fosse eliminada, estima-se uma redução de cerca de 8,2\% de doenças coronarianas e $13 \%$ de mortes por causas gerais ${ }^{1}$. No entanto, a pre- 
valência de atividade física suficiente no Brasil ainda é baixa, sendo que dados da Pesquisa Nacional de Saúde (PNS), realizada em 2013, mostraram que a prevalência de pessoas ativas no lazer (praticavam pelo menos 150 minutos de atividade física leve ou moderada, ou pelo menos 75 minutos de atividade física vigorosa, ou então a combinação de atividades moderadas e vigorosas que somassem 150 minutos semanais no lazer) era de $22,5 \%$, sendo maior em homens $(27,1 \%)^{2}$.

Outro comportamento que vem sendo considerado no combate às Doenças Crônicas Não Transmissíveis (DCNTs) é o comportamento sedentário (CS), definido como atividades sentadas ou deitadas que demandam baixos níveis de gasto energético, abaixo de 1,5 MET (equivalente metabólico da tarefa) ${ }^{3}$. Estudos demonstram que o comportamento sedentário é associado com doenças crônicas independentemente dos níveis de atividade física ${ }^{4,5}$. Similarmente a atividade física, estima-se que cerca de $2,5 \%$ da mortalidade por causas gerais seriam evitadas no Brasil, caso o comportamento sedentário fosse erradicado ${ }^{6}$. Diversos estudos têm indicado a associação do CS, especialmente o hábito de assistir televisão, com morbidade e mortalidade por $\mathrm{DCNTs}^{7,9}$.

Inquéritos nacionais de saúde fornecem informações importantes sobre indicadores e tendências acerca da saúde da população ao longo do tempo ${ }^{10-12}$. Pesquisas com tal abrangência destacam-se pela capacidade de mensurar os fatores de risco modificáveis para doenças crônicas, sendo relevantes no direcionamento de futuras intervenções e planejamento de políticas públicas em saúde ${ }^{10-12}$. No Brasil, o Plano de Ações Estratégicas para o Enfrentamento das Doenças Crônicas Não Transmissíveis (DCNTs) 2011-2022 visa, dentre outros objetivos, modificar os fatores de risco que têm relevância para a prevenção das DCNTs. Dentre as ações compreendidas por este plano, está o aumento do nível de atividade física da população ${ }^{13}$.

Nesse contexto, o Sistema de Vigilância de Fatores de Risco e Proteção para Doenças Crônicas por Inquérito Telefônico (VIGITEL) e a PNS são dois inquéritos brasileiros que tem investigado sobre os níveis de atividade física e os hábitos de comportamento sedentário na população. Entretanto, diferenciam quanto à temporalidade, abrangência, amostragem e o método de aplicação dos questionários. O VIGITEL é realizado anualmente por meio de entrevistas telefônicas abrangendo as capitais do país, enquanto a PNS foi realizada por meio de questionários aplicados face-a-face em amostra representativa do Brasil ${ }^{14,15}$. A condução da PNS demanda logística mais complexa e custos mais elevados em relação ao VIGITEL ${ }^{12}$. Inquéritos telefônicos tendem a obter informações semelhantes ao inquérito domiciliar realizado face-a-face ${ }^{16}$, e constituem um método de baixo custo e agilidade na consolidação, análise e divulgação dos seus resultados. Entretanto, a cobertura limitada de linha telefônica fixa pode interferir na qualidade dos seus dados ${ }^{17}$.

Inquéritos telefônicos tendem a obter informações semelhantes ao inquérito domiciliar realizado face-a-face ${ }^{11}$. Entretanto, a qualidade dos dados no inquérito por telefone pode ser influenciada pela limitada cobertura de linha telefônica fixa ${ }^{17}$. Por outro lado, os inquéritos por telefone constituem um método com custo e tempo de coleta menores ${ }^{10}$. Considerando que a condução da PNS demanda logística mais complexa e custos mais elevados em relação ao VIGITEL ${ }^{12}$, o objetivo deste estudo foi comparar as estimativas de atividade física e comportamento entre o VIGITEL e PNS realizados em 2013.

\section{Métodos}

Foi realizado um estudo de comparação entre dois estudos epidemiológicos brasileiros, utilizando dados secundários do VIGITEL e da PNS coletados no ano de 2013. O VIGITEL é um inquérito telefônico que ocorre anualmente desde 2006, nas 26 capitais brasileiras e no Distrito Federal. A população pesquisada é composta por adultos com 18 anos de idade ou mais, residentes em domicílios servidos por pelo menos uma linha telefônica fixa. Em cada capital, a amostragem é realizada primeiramente por meio de seleção randomizada das linhas telefônicas fixas, com base no cadastro eletrônico de empresas telefônicas, sendo sorteadas 5.000 linhas por cidade estratificadas por Código de Endereçamento Postal (CEP) e após, são feitas subamostras de 200 linhas cada para a identificação de linhas elegíveis (residenciais e ativas). Posteriormente, em cada linha que houve contato e o morador adulto concordou em participar, ocorre de forma aleatória a seleção do indivíduo no domicílio a ser entrevistado (apenas um por residência). O sistema estabelece um tamanho amostral mínimo de aproximadamente dois mil indivíduos em cada capital e no Distrito Federal. Mais detalhes sobre a metodologia utilizada nesse inquérito estão disponíveis em outra publicação ${ }^{15}$.

A PNS foi um inquérito domiciliar, realizado em 2013 com moradores de domicílios particulares perma- 
nentes de todo o território nacional, sendo excluídos os setores especiais (quartéis, bases militares, alojamentos, acampamentos, embarcações, penitenciárias, colônias penais, presídios, cadeias, asilos, orfanatos, conventos e hospitais). A amostragem foi realizada por conglomerados, sendo as unidades primárias de amostragem formadas pelos setores censitários ou conjunto de setores, as unidades secundárias foram compostas pelos domicílios e a unidade terciária formada por moradores com 18 anos ou mais de idade. Os setores censitários foram sorteados com base no Sistema Integrado de Pesquisas Domiciliares (SIPD) do Instituto Brasileiro de Geografia e Estatística (IBGE), e utiliza a amostra mestra desse sistema, a qual é caracterizada por um conjunto de áreas selecionadas para atender diversas pesquisas, proporcionando maior expansão geográfica e melhor precisão das estimativas. Foram conduzidas três entrevistas com o morador, sendo: 1) informações sobre características do domicílio, 2) informações referentes a todos os moradores a partir do relato de um informante do domicílio, e 3) informações referentes a um morador de 18 anos ou mais do domicílio, selecionado aleatoriamente a partir da lista de moradores obtida no momento da entrevista. A metodologia detalhada desse inquérito está disponível no documento com os resultados da PNS de 2013, bem como por Szwarcwald et al. ${ }^{18} \mathrm{e}$ o processo de amostragem detalhado encontra-se em publicação prévia ${ }^{19}$.

Os dados analisados neste estudo referem-se à entrevista individual, tendo em vista que para os demais moradores não foram coletadas as informações sobre atividade física e tempo de televisão. Além da amostra total da PNS, uma sub-amostra considerando apenas as capitais dos estados foi analisada. Todos os procedimentos dos inquéritos foram aprovados pela Comissão Nacional de Ética em Pesquisa, de acordo com a declaração de Helsinki (PNS - processo 10853812.7.0000.0008; VIGITEL - processo CONEP 355590).

Os desfechos utilizados foram escolhidos a partir da comparabilidade entre as perguntas utilizadas nos inquéritos ${ }^{13,14}$, embora ainda exista alguma diferença entre as questões utilizadas no presente estudo, selecionadas a partir de uma lista de aproximadamente 20 questões sobre o assunto. As principais diferenças encontram-se nas opções de respostas, sendo que no VIGITEL o indivíduo precisa escolher uma das categorias referente aos dias e tempo de prática de atividade física realizada, enquanto na PNS as respostas são abertas. Sendo assim, foram analisados quatro desfechos: indivíduos suficientemente ativos no lazer, deslocamento ativo para o trabalho, tempo de TV maior ou igual a três horas e inatividade física no lazer. Foram considerados suficientemente ativos no lazer os indivíduos que realizaram exercício físico e/ou esporte em no mínimo cinco dias por semana durante 30 minutos por dia, considerando que a duração semanal não poderia ser calculada a partir das alternativas de resposta do VIGITEL. O deslocamento ativo foi caracterizado pela realização do trajeto ao trabalho a pé ou de bicicleta. A inatividade física no lazer foi definida pela ausência de prática de qualquer exercício físico ou esporte nos últimos três meses. Com relação ao tempo de televisão, analisou-se a proporção de indivíduos que passava em média três horas ou mais assistindo televisão, conforme critério utilizado ${ }^{6,13,14}$.

As variáveis independentes utilizadas no estudo foram: sexo (masculino; feminino), idade (18-39 anos; 40-59 anos; 60 anos ou mais), escolaridade (sem instrução; 1-8 anos; 9-11 anos; 12 anos ou mais) e IMC, como indicador de saúde e nutrição, calculado a partir do auto relato de peso e altura e definido conforme os critérios da Organização Mundial de Saúde (OMS) ${ }^{20}$ (baixo peso ou eutrófico - até $24,9 \mathrm{~kg} / \mathrm{m} 2$; sobrepeso 25,0 a $29,9 \mathrm{~kg} / \mathrm{m}^{2}$; obeso - 30,0 ou mais $\mathrm{kg} / \mathrm{m}^{2}$ ). Foram utilizados os valores de IMC autorreferidos.

Realizou-se as análises considerando-se a base completa, bem como somente as capitais. Para a comparação da ocorrência dos desfechos entre os dois inquéritos, foram analisadas a concordância e a diferença em pontos percentuais. A análise de concordância foi realizada por meio do Coeficiente de Correlação de Concordância de Lin $(\mathrm{CCC})^{21}$, tendo os estados brasileiros e também apenas as capitais como unidade amostral. O CCC pode ser interpretado como o produto das medidas de correlação de Pearson e acurácia - a concordância perfeita equivale a $\mathrm{CCC}=1,0$. Foram calculadas as diferenças em pontos percentuais considerando as proporções nas duas amostras, sendo a diferença estatística observada pela não sobreposição dos intervalos de confiança de $95 \%$. Todas as análises foram realizadas considerando os pesos amostrais definidos em cada inquérito a partir do comando svy e calculadas as proporções dos desfechos conforme o inquérito e as variáveis independentes com seus respectivos intervalos de confiança de $95 \%$. Foi utilizado o pacote estatístico Stata 13.0. 


\section{Resultados}

As amostras dos inquéritos VIGITEL e PNS realizados durante o ano de 2013, compostas respectivamente por 52.929 e 60.202 entrevistados, foram comparadas de acordo com as variáveis independentes descritas na Tabela 1. Considerando as amostras totais (Diferença 1-2), as principais diferenças entre os inquéritos ocorreram na distribuição de escolaridade e IMC. Em relação à escolaridade, apenas $2,0 \%$ dos entrevistados no VIGITEL não tinham instrução, enquanto $13,7 \%$ dos entrevistados na PNS declararam-se sem instrução, com diferença estatisticamente significativa de $-11,7$ pontos percentuais (p.p.) (IC95\%: -12,8; -10,6). Com relação ao IMC, a maioria dos entrevistados, em ambos inquéritos, pertencia à categoria baixo peso ou eutrófico. Porém, no VIGITEL foram entrevistados mais indivíduos pertencentes a essa categoria em comparação com a PNS. Ao contrário, os indivíduos com sobrepeso ou obesidade foram mais prevalentes na PNS, porém as diferenças entre os inquéritos foram menores.

Os indivíduos suficientemente ativos no lazer foram comparados entre os dois inquéritos de acordo com as variáveis independentes, como mostrado na Tabela 2 . A frequência de indivíduos considerados suficientemente ativos pelo VIGITEL foi estatisticamente superior às frequências da PNS para todas as categorias das variáveis independentes. $\mathrm{O}$ mesmo padrão foi observado quando o VIGITEL foi comparado à sub-amostra das capitais da PNS, porém as diferenças foram ainda maiores para todas as variáveis analisadas. As estimativas do VIGITEL e da PNS foram similares com relação ao transporte ativo para o trabalho. Porém, foram observadas grande variabilidade entre as estimativas, uma vez que o VIGITEL ora produziu estimativas superiores às da PNS, e ora o contrário. Entretanto, comparando com a sub-amostra de capitais da PNS as estimativas do VIGITEL superestimaram os indivíduos que realizam o deslocamento ativo para o trabalho.

Tabela 1 - Distribuição das amostras do VIGITEL 2013 ( $\mathrm{N}=52.929)$, PNS 2013 ( $\mathrm{N}=60.202)$ e PNS 2013 considerando apenas capitais $(\mathrm{N}=27.017)$.

\begin{tabular}{|c|c|c|c|c|c|}
\hline \multirow{2}{*}{ Variáveis } & $\begin{array}{l}\text { VIGITEL } \\
\text { (1) }\end{array}$ & $\begin{array}{l}\text { PNS } \\
(2)\end{array}$ & $\begin{array}{l}\text { PNS Cap. } \\
\text { (3) }\end{array}$ & $\begin{array}{c}\text { Diferença } \\
(1-2)\end{array}$ & $\begin{array}{c}\text { Diferença } \\
(1-3)\end{array}$ \\
\hline & $\begin{array}{c}\mathrm{N} \\
\%(\mathrm{IC} 95 \%)\end{array}$ & $\begin{array}{c}\mathrm{N} \\
\%(\mathrm{IC} 95 \%)\end{array}$ & $\begin{array}{c}\mathrm{N} \\
\%(\mathrm{IC} 95 \%)\end{array}$ & p.p. & p.p. \\
\hline \multicolumn{6}{|l|}{ Sexo } \\
\hline Masculino & $\begin{array}{c}20.276 \\
46,1(45,2-46,9)\end{array}$ & $\begin{array}{c}25.920 \\
47,1(46,4-47,8)\end{array}$ & $\begin{array}{c}11.091 \\
45,1(44,1-46,0)\end{array}$ & $-1,0^{*}$ & 1,0 \\
\hline Feminino & $\begin{array}{c}32.653 \\
53,9(53,1-54,8)\end{array}$ & $\begin{array}{c}34.282 \\
52,9(52,2-53,6)\end{array}$ & $\begin{array}{c}15.926 \\
54,9(54,0-55,9)\end{array}$ & $1,0^{*}$ & $-0,8$ \\
\hline \multicolumn{6}{|l|}{ Idade (anos) } \\
\hline $18-39$ & $\begin{array}{c}18.325 \\
50,3(49,4-5,1)\end{array}$ & $\begin{array}{c}28.590 \\
47,7(47,0-48,4)\end{array}$ & $\begin{array}{c}12.953 \\
48,0(47,1-48,9)\end{array}$ & $2,6^{*}$ & $2,3^{*}$ \\
\hline $40-59$ & $\begin{array}{c}19.622 \\
33,6(32,8-34,4)\end{array}$ & $\begin{array}{c}20.435 \\
34,2(33,6-34,9)\end{array}$ & $\begin{array}{c}9.152 \\
34,0(33,1-34,8)\end{array}$ & $-0,6$ & $-0,4$ \\
\hline $60+$ & $\begin{array}{c}14.982 \\
16,1(15,6-16,7)\end{array}$ & $\begin{array}{c}11.177 \\
18,1(17,5-18,6)\end{array}$ & $\begin{array}{c}4.912 \\
18,0(17,4-18,7)\end{array}$ & $-2,0^{*}$ & $-1,9^{*}$ \\
\hline \multicolumn{6}{|l|}{ Escolaridade (anos) } \\
\hline 0 & $\begin{array}{c}1.146 \\
2,0(1,8-2,3)\end{array}$ & $\begin{array}{c}9.434 \\
13,7(13,2-14,2)\end{array}$ & $\begin{array}{c}2.692 \\
8,0(7,6-8,5)\end{array}$ & $-11,7^{*}$ & $-6,0^{*}$ \\
\hline $1-8$ & $\begin{array}{c}13.641 \\
34,6(33,7-35,5)\end{array}$ & $\begin{array}{c}20.537 \\
35,2(34,5-35,8)\end{array}$ & $\begin{array}{c}7.505 \\
27,0(26,2-27,8)\end{array}$ & $-0,6$ & $7,6^{*}$ \\
\hline $9-11$ & $\begin{array}{c}19.636 \\
37,5(36,6-38,2)\end{array}$ & $\begin{array}{c}19.438 \\
33,6(33,0-34,3)\end{array}$ & $\begin{array}{c}9.868 \\
38,1(37,2-39,0)\end{array}$ & $3,9^{*}$ & $-0,6$ \\
\hline $12+$ & $\begin{array}{c}18.506 \\
25,9(25,3-26,7)\end{array}$ & $\begin{array}{c}10.793 \\
17,5(17,0-18,1)\end{array}$ & $\begin{array}{c}6.952 \\
26,9(26,1-27,7)\end{array}$ & $8,4^{*}$ & $-1,0$ \\
\hline \multicolumn{6}{|l|}{$\operatorname{IMC}\left(\mathrm{kg} / \mathrm{m}^{2}\right)$} \\
\hline Baixo peso ou eutrófico & $\begin{array}{c}22.709 \\
48,9(48,0-49,8)\end{array}$ & $\begin{array}{c}18.793 \\
45,8(45,0-46,7)\end{array}$ & $\begin{array}{c}9.264 \\
45,4(44,3-46,4)\end{array}$ & $3,1^{*}$ & $3,5^{*}$ \\
\hline Sobrepeso & $\begin{array}{c}16.974 \\
33,7(32,9-34,6)\end{array}$ & $\begin{array}{c}14.589 \\
35,5(34,7-36,3)\end{array}$ & $\begin{array}{c}7.288 \\
36,2(35,2-37,3)\end{array}$ & $-1,8^{*}$ & $-2,5^{*}$ \\
\hline Obeso & $\begin{array}{c}8.582 \\
17,4(16,7-18,1)\end{array}$ & $\begin{array}{c}7.560 \\
18,7(18,0-19,4)\end{array}$ & $\begin{array}{c}3.787 \\
18,4(17,6-19,3)\end{array}$ & $-1,3^{*}$ & $-1,0$ \\
\hline
\end{tabular}

Cap. $=$ capitais IMC $=$ índice de massa corporal; p.p. = pontos percentuais; ${ }^{*} \mathrm{p}<0,05$. 
Tabela 2 - Percentual de adultos ativos no lazer e que realizam deslocamento ativo para o trabalho de acordo com sexo, idade, escolaridade e estado nutricional e inquérito (VIGITEL, PNS e PNS somente capitais), 2013

\begin{tabular}{|c|c|c|c|c|c|c|c|c|c|c|}
\hline \multirow{3}{*}{ Variáveis } & \multicolumn{5}{|c|}{ Deslocamento ativo para trabalho } & \multicolumn{5}{|c|}{ Deslocamento ativo para trabalho } \\
\hline & $\begin{array}{l}\text { VIGITEL } \\
\text { (1) }\end{array}$ & $\begin{array}{l}\text { PNS } \\
(2)\end{array}$ & $\begin{array}{l}\text { PNS Cap. } \\
\text { (3) }\end{array}$ & $\begin{array}{c}\text { Diferença } \\
(1-2)\end{array}$ & $\begin{array}{c}\text { Diferença } \\
(1-3)\end{array}$ & $\begin{array}{l}\text { VIGITEL } \\
\text { (4) }\end{array}$ & $\begin{array}{l}\text { PNS } \\
(5)\end{array}$ & $\begin{array}{l}\text { PNS Cap. } \\
\text { (6) }\end{array}$ & $\begin{array}{c}\text { Diferença } \\
(4-5)\end{array}$ & $\begin{array}{c}\text { Diferença } \\
(4-6)\end{array}$ \\
\hline & $\begin{array}{c}\% \\
\text { (IC95\%) }\end{array}$ & $\begin{array}{c}\% \\
\text { (IC95\%) }\end{array}$ & $\begin{array}{c}\% \\
\text { (IC95\%) }\end{array}$ & p.p. & p.p. & $\begin{array}{c}\% \\
\text { (IC95\%) }\end{array}$ & $\begin{array}{c}\% \\
\text { (IC95\%) }\end{array}$ & $\begin{array}{c}\% \\
\text { (IC95\%) }\end{array}$ & p.p. & p.p. \\
\hline \multicolumn{11}{|l|}{ Sexo } \\
\hline Masculino & $\begin{array}{c}16,4 \\
(15,5-17,4)\end{array}$ & $\begin{array}{c}3,7 \\
(3,3-4,2)\end{array}$ & $\begin{array}{c}5,2 \\
(4,6-5,9)\end{array}$ & $12,7^{*}$ & $11,2^{*}$ & $\begin{array}{c}36,8 \\
(35,2-38,4)\end{array}$ & $\begin{array}{c}36,4 \\
(35,2-37,6)\end{array}$ & $\begin{array}{c}35,6 \\
(34,0-37,2)\end{array}$ & 0,4 & $1,2^{*}$ \\
\hline Feminino & $\begin{array}{c}13,5 \\
(12,8-14,3)\end{array}$ & $\begin{array}{c}3,9 \\
(3,6-4,4)\end{array}$ & $\begin{array}{c}4,0 \\
(3,6-4,5)\end{array}$ & $9,6^{*}$ & $9,5^{*}$ & $\begin{array}{c}45,5 \\
(44,0-47,1)\end{array}$ & $\begin{array}{c}46,3 \\
(45,0-47,7)\end{array}$ & $\begin{array}{c}40,8 \\
(39,2-42,4)\end{array}$ & $-0,8^{*}$ & $4,7^{*}$ \\
\hline \multicolumn{11}{|l|}{ Idade (anos) } \\
\hline $18-39$ & $\begin{array}{c}17,7 \\
(16,7-18,7)\end{array}$ & $\begin{array}{c}4,1 \\
(3,7-4,6)\end{array}$ & $\begin{array}{c}4,9 \\
(4,4-5,6)\end{array}$ & $13,6^{*}$ & $12,8^{*}$ & $\begin{array}{c}41,8 \\
(40,3-43,4)\end{array}$ & $\begin{array}{c}39,9 \\
(38,7-41,1)\end{array}$ & $\begin{array}{c}39,9 \\
(38,3-41,4)\end{array}$ & $1,9^{*}$ & $1,9^{*}$ \\
\hline $40-59$ & $\begin{array}{c}11,9 \\
(11,1-12,7)\end{array}$ & $\begin{array}{c}3,3 \\
(2,9-3,8)\end{array}$ & $\begin{array}{c}4,1 \\
(3,5-4,8)\end{array}$ & $8,6^{*}$ & $7,8^{*}$ & $\begin{array}{c}39,9 \\
(38,2-41,7)\end{array}$ & $\begin{array}{c}41,9 \\
(40,5-43,3)\end{array}$ & $\begin{array}{c}36,5 \\
(34,7-38,3)\end{array}$ & $-2,0$ & 3,4 \\
\hline $60+$ & $\begin{array}{c}12,2 \\
(11,2-13,3)\end{array}$ & $\begin{array}{c}4,1 \\
(3,5-4,8)\end{array}$ & $\begin{array}{c}4,5 \\
(3,7-5,4)\end{array}$ & $8,1^{*}$ & $7,7^{*}$ & $\begin{array}{c}36,0 \\
(32,6-39,6)\end{array}$ & $\begin{array}{c}39,6 \\
(36,3-43,1)\end{array}$ & $\begin{array}{c}31,2 \\
(27,3-35,4)\end{array}$ & $-3,6$ & 4,8 \\
\hline \multicolumn{11}{|c|}{ Escolaridade (anos) } \\
\hline 0 & $\begin{array}{c}7,0 \\
(4,8-10,3)\end{array}$ & $\begin{array}{c}2,0 \\
(1,6-2,6)\end{array}$ & $\begin{array}{c}2,1 \\
(1,4-3,0)\end{array}$ & $5,0^{*}$ & $4,9^{*}$ & $\begin{array}{c}49,8 \\
(37,5-62,2)\end{array}$ & $\begin{array}{c}52,7 \\
(49,8-55,5)\end{array}$ & $\begin{array}{c}50,0 \\
(45,5-54,6)\end{array}$ & $-2,9$ & $-0,2$ \\
\hline $1-8$ & $\begin{array}{c}10,4 \\
(9,5-11,4)\end{array}$ & $\begin{array}{c}2,7 \\
(2,3-3,1)\end{array}$ & $\begin{array}{c}2,5 \\
(2,1-3,1)\end{array}$ & $7,7^{*}$ & $7,9^{*}$ & $\begin{array}{c}43,4 \\
(41,1-45,9)\end{array}$ & $\begin{array}{c}48,1 \\
(46,4-49,7)\end{array}$ & $\begin{array}{c}44,8 \\
(42,4-47,2)\end{array}$ & $-4,7^{*}$ & $-1,4$ \\
\hline $9-11$ & $\begin{array}{c}16,1 \\
(15,1-17,0)\end{array}$ & $\begin{array}{c}4,8 \\
(4,2-5,4)\end{array}$ & $\begin{array}{c}5,0 \\
(4,3-5,8)\end{array}$ & $11,3^{*}$ & $11,1^{*}$ & $\begin{array}{c}42,8 \\
(41,1-44,5)\end{array}$ & $\begin{array}{c}39,3 \\
(37,8-40,8)\end{array}$ & $\begin{array}{c}40,7 \\
(38,9-42,6)\end{array}$ & $3,5^{*}$ & $2,1^{*}$ \\
\hline $12+$ & $\begin{array}{c}19,5 \\
(18,3-20,8)\end{array}$ & $\begin{array}{c}5,8 \\
(5,1-6,7)\end{array}$ & $\begin{array}{c}6,7 \\
(5,9-7,7)\end{array}$ & $13,7^{*}$ & $12,8^{*}$ & $\begin{array}{c}35,4 \\
(33,6-37,3)\end{array}$ & $\begin{array}{c}27,6 \\
(25,9-29,3)\end{array}$ & $\begin{array}{c}27,9 \\
(26,0-29,9)\end{array}$ & $7,8^{*}$ & $7,5^{*}$ \\
\hline \multicolumn{11}{|l|}{$\operatorname{IMC}\left(\mathrm{kg} / \mathrm{m}^{2}\right)$} \\
\hline $\begin{array}{l}\text { Baixo peso ou } \\
\text { eutrófico }\end{array}$ & $\begin{array}{c}15,5 \\
(14,7-16,4)\end{array}$ & $\begin{array}{c}4,3 \\
(3,8-4,9)\end{array}$ & $\begin{array}{c}5,2 \\
(4,5-5,9)\end{array}$ & $11,2^{*}$ & $10,3^{*}$ & $\begin{array}{c}43,6 \\
(41,9-45,3)\end{array}$ & $\begin{array}{c}42,0 \\
(40,4-43,5)\end{array}$ & $\begin{array}{c}39,9 \\
(38,0-41,9)\end{array}$ & $1,6^{*}$ & $3,7^{*}$ \\
\hline Sobrepeso & $\begin{array}{c}17,2 \\
(16,0-18,4)\end{array}$ & $\begin{array}{c}5,0 \\
(4,3-5,6)\end{array}$ & $\begin{array}{c}5,9 \\
(5,1-6,9)\end{array}$ & $12,2^{*}$ & $11,3^{*}$ & $\begin{array}{c}37,9 \\
(36,0-39,8)\end{array}$ & $\begin{array}{c}35,1 \\
(33,5-36,8)\end{array}$ & $\begin{array}{c}34,4 \\
(32,3-36,4)\end{array}$ & $2,8^{*}$ & $3,5^{*}$ \\
\hline Obeso & $\begin{array}{c}12,8 \\
(10,6-13,2)\end{array}$ & $\begin{array}{c}3,4 \\
(2,8-4,2)\end{array}$ & $\begin{array}{c}3,7 \\
(2,9-4,8)\end{array}$ & $9,4^{*}$ & $9,1^{*}$ & $\begin{array}{c}34,2 \\
(31,5-37,0)\end{array}$ & $\begin{array}{c}34,2 \\
(31,9-36,6)\end{array}$ & $\begin{array}{c}34,0 \\
(31,1-37,0)\end{array}$ & 0,0 & 0,2 \\
\hline
\end{tabular}

Cap. $=$ capitais IMC $=$ índice de massa corporal; p.p. = pontos percentuais; ${ }^{*} \mathrm{p}<0,05$.

Na Tabela 3 são comparados os desfechos referentes ao tempo sedentário e inatividade no lazer. As estimativas de tempo de TV superior ou igual a três horas por dia foram significativamente diferentes entre o VIGITEL e a PNS conforme sexo e escolaridade. Os resultados diferiram quando comparou-se o VIGITEL com a sub-amostra de capitais da PNS. O percentual de indivíduos que apresentaram tempo de TV maior ou igual a três horas foi significativamente superior na PNS capitais para todas as categorias das variáveis independentes. As maiores diferenças entre as estimativas dos inquéritos foram encontradas com relação à inatividade física no lazer, onde a PNS gerou estimativas consistentemente superiores às do VIGITEL (Figura 1C).

As estimativas de prevalência de indivíduos suficientemente ativos no lazer apresentaram concordância baixa. O CCC foi igual a 0,011 na comparação do VI-
GITEL com a amostra total da PNS e 0,028 na comparação com a PNS Capitais (Figura 1 A e B). Porém, ao observar as prevalências de realização de deslocamento ativo para o trabalho (Figura $1 \mathrm{C} \mathrm{e} \mathrm{D),} \mathrm{percebe-se} \mathrm{con-}$ cordância de moderada à alta entre as estimativas dos inquéritos para cada estado brasileiro $(\mathrm{CCC}=0,516$ considerando toda a amostra da PNS, e CCC $=0,721$ para PNS Capitais). Além disso, a linha reflete uma relação próxima da linear entre as estimativas dos inquéritos. Apesar disso, alguns resultados foram bastante discrepantes entre os inquéritos, a exemplo do estado do Tocantins, que teve sua prevalência de deslocamento ativo muito inferior no VIGITEL quando comparada à estimativa obtida pela PNS (23,9\% vs. $41,6 \%)$.

Considerando o tempo de TV, foi considerado moderado na comparação do VIGITEL com a amostra total da PNS $(\mathrm{CCC}=0,412)$ e baixo na comparação 
Tabela 3 - Percentual de indivíduos com tempo de TV maior ou igual a 3 horas e considerados inativos no lazer de acordo com variáveis independentes e inquérito, 2013.

\begin{tabular}{|c|c|c|c|c|c|c|c|c|c|c|}
\hline \multirow{3}{*}{ Variáveis } & \multicolumn{5}{|c|}{ Deslocamento ativo para trabalho } & \multicolumn{5}{|c|}{ Inatividade no lazer } \\
\hline & $\begin{array}{l}\text { VIGITEL } \\
\text { (1) }\end{array}$ & $\begin{array}{l}\text { PNS } \\
\text { (2) }\end{array}$ & $\begin{array}{l}\text { PNS Cap. } \\
\text { (3) }\end{array}$ & $\begin{array}{c}\text { Diferença } \\
(1-2)\end{array}$ & $\begin{array}{c}\text { Diferença } \\
(1-3)\end{array}$ & $\begin{array}{l}\text { VIGITEL } \\
\text { (4) }\end{array}$ & $\begin{array}{l}\mathrm{PNS} \\
(5)\end{array}$ & $\begin{array}{l}\text { PNS Cap. } \\
\text { (6) }\end{array}$ & $\begin{array}{c}\text { Diferença } \\
(4-5)\end{array}$ & $\begin{array}{c}\text { Diferença } \\
(4-6)\end{array}$ \\
\hline & $\begin{array}{c}\% \\
(\mathrm{IC} 95 \%)\end{array}$ & $\begin{array}{c}\% \\
(\mathrm{IC} 95 \%)\end{array}$ & $\begin{array}{c}\% \\
\text { (IC95\%) }\end{array}$ & p.p. & p.p. & $\begin{array}{c}\% \\
\text { (IC95\%) }\end{array}$ & $\begin{array}{c}\% \\
(\mathrm{IC} 95 \%)\end{array}$ & $\begin{array}{c}\% \\
\text { (IC95\%) }\end{array}$ & p.p. & p.p. \\
\hline \multicolumn{11}{|l|}{ Sexo } \\
\hline Masculino & $\begin{array}{c}28,1 \\
(26,8-29,4)\end{array}$ & $\begin{array}{c}25,5 \\
(24,6-26,5)\end{array}$ & $\begin{array}{c}30,2 \\
(28,9-31,6)\end{array}$ & $2,6^{*}$ & $-2,1^{*}$ & $\begin{array}{c}45,0 \\
(43,6-46,4)\end{array}$ & $\begin{array}{c}63,1 \\
(62,1-64,2)\end{array}$ & $\begin{array}{c}56,7 \\
(55,2-58,1)\end{array}$ & $-18,1^{*}$ & $-11,7^{*}$ \\
\hline Feminino & $\begin{array}{c}29,0 \\
(28,0-30,0)\end{array}$ & $\begin{array}{c}31,9 \\
(31,1-32,8)\end{array}$ & $\begin{array}{c}35,2 \\
(34,1-36,4)\end{array}$ & $-2,9^{*}$ & $6,2^{*}$ & $\begin{array}{c}59,5 \\
(58,4-60,6)\end{array}$ & $\begin{array}{c}73,2 \\
(72,3-74,0)\end{array}$ & $\begin{array}{c}69,9 \\
(68,8-71,0)\end{array}$ & $-13,7^{*}$ & $-10,4^{*}$ \\
\hline \multicolumn{11}{|l|}{ Idade (anos) } \\
\hline $18-39$ & $\begin{array}{c}28,2 \\
(27,0-29,5)\end{array}$ & $\begin{array}{c}29,4 \\
(28,4-30,3)\end{array}$ & $\begin{array}{c}31,5 \\
(30,3-32,8)\end{array}$ & $-1,2^{*}$ & $-3,3^{*}$ & $\begin{array}{c}45,7 \\
(44,4-47,0)\end{array}$ & $\begin{array}{c}61,2 \\
(60,2-62,2)\end{array}$ & $\begin{array}{c}58,3 \\
(57,0-59,6)\end{array}$ & $-15,5^{*}$ & $-12,6^{*}$ \\
\hline $40-59$ & $\begin{array}{c}26,8 \\
(25,5-28,0)\end{array}$ & $\begin{array}{c}26,4 \\
(25,4-27,5)\end{array}$ & $\begin{array}{c}30,7 \\
(29,3-32,2)\end{array}$ & $0,4^{*}$ & $-3,9^{*}$ & $\begin{array}{c}59,0 \\
(57,7-60,4)\end{array}$ & $\begin{array}{c}73,4 \\
(72,4-74,5)\end{array}$ & $\begin{array}{c}67,0 \\
(65,5-68,5)\end{array}$ & $-14,4^{*}$ & $-8,0^{*}$ \\
\hline $60+$ & $\begin{array}{c}33,5 \\
(31,9-35,2)\end{array}$ & $\begin{array}{c}32,4 \\
(30,9-33,9)\end{array}$ & $\begin{array}{c}41,1 \\
(39,1-43,2)\end{array}$ & $1,1^{*}$ & $-7,6^{*}$ & $\begin{array}{c}62,3 \\
(60,5-63,8)\end{array}$ & $\begin{array}{c}78,2 \\
(76,8-79,5)\end{array}$ & $\begin{array}{c}73,2 \\
(71,3-75,0)\end{array}$ & $-15,9^{*}$ & $-10,9^{*}$ \\
\hline \multicolumn{11}{|c|}{ Escolaridade (anos) } \\
\hline 0 & $\begin{array}{c}20,8 \\
(16,6-25,8)\end{array}$ & $\begin{array}{c}26,5 \\
(25,0-28,1)\end{array}$ & $\begin{array}{c}35,7 \\
(33,0-38,5)\end{array}$ & $-5,7^{*}$ & $-14,9^{*}$ & $\begin{array}{c}81,4 \\
(76,6-85,4)\end{array}$ & $\begin{array}{c}85,9 \\
(84,6-87,1)\end{array}$ & $\begin{array}{c}85,1 \\
(82,9-87,1)\end{array}$ & $-4,5^{*}$ & $-3,7^{*}$ \\
\hline $1-8$ & $\begin{array}{c}32,0 \\
(30,5-33,6)\end{array}$ & $\begin{array}{c}30,4 \\
(29,3-31,5)\end{array}$ & $\begin{array}{c}37,8 \\
(36,2-39,5)\end{array}$ & $1,6^{*}$ & $-5,8^{*}$ & $\begin{array}{c}65,2 \\
(63,7-66,7)\end{array}$ & $\begin{array}{c}77,0 \\
(76,0-78,1)\end{array}$ & $\begin{array}{c}77,6 \\
(76,1-79,0)\end{array}$ & $-11,8^{*}$ & $-12,4^{*}$ \\
\hline $9-11$ & $\begin{array}{c}30,6 \\
(29,4-31,9)\end{array}$ & $\begin{array}{c}32,4 \\
(31,2-33,5)\end{array}$ & $\begin{array}{c}35,9 \\
(34,5-37,4)\end{array}$ & $-1,8^{*}$ & $-5,3^{*}$ & $\begin{array}{c}50,2 \\
(48,8-51,5)\end{array}$ & $\begin{array}{c}62,5 \\
(61,3-63,7)\end{array}$ & $\begin{array}{c}62,8 \\
(61,3-64,3)\end{array}$ & $-12,3^{*}$ & $-12,6^{*}$ \\
\hline $12+$ anos & $\begin{array}{c}21,6 \\
(20,4-22,9)\end{array}$ & $\begin{array}{c}21,2 \\
(19,9-22,6)\end{array}$ & $\begin{array}{c}23,2 \\
(21,7-24,7)\end{array}$ & 0,4 & $-1,6$ & $\begin{array}{c}37,9 \\
(36,4-39,5)\end{array}$ & $\begin{array}{c}49,0 \\
(47,3-50,7)\end{array}$ & $\begin{array}{c}45,5 \\
(43,7-47,2)\end{array}$ & $-11,1^{*}$ & $-7,6^{*}$ \\
\hline \multicolumn{11}{|l|}{$\operatorname{IMC}\left(\mathrm{kg} / \mathrm{m}^{2}\right)$} \\
\hline $\begin{array}{l}\text { Baixo peso ou } \\
\text { eutrófico }\end{array}$ & $\begin{array}{c}27,0 \\
(25,9-28,2)\end{array}$ & $\begin{array}{c}26,3 \\
(25,2-27,4)\end{array}$ & $\begin{array}{c}29,1 \\
(27,7-30,5)\end{array}$ & $0,7^{*}$ & $-2,1^{*}$ & $\begin{array}{c}50,1 \\
(48,8-51,4)\end{array}$ & $\begin{array}{c}62,3 \\
(61,0-63,5)\end{array}$ & $\begin{array}{c}57,7 \\
(56,1-59,2)\end{array}$ & $-12,2^{*}$ & $-7,6^{*}$ \\
\hline Sobrepeso & $\begin{array}{c}28,8 \\
(27,5-30,2)\end{array}$ & $\begin{array}{c}28,6 \\
(27,3-29,9)\end{array}$ & $\begin{array}{c}32,1 \\
(30,5-33,8)\end{array}$ & $0,2^{*}$ & $-3,3^{*}$ & $\begin{array}{c}48,8 \\
(47,3-50,3)\end{array}$ & $\begin{array}{c}61,6 \\
(60,2-63,0)\end{array}$ & $\begin{array}{c}57,8 \\
(56,0-59,5)\end{array}$ & $-12,8^{*}$ & $-9,0^{*}$ \\
\hline Obeso & $\begin{array}{c}32,4 \\
(30,3-34,4)\end{array}$ & $\begin{array}{c}32,7 \\
(30,8-34,6)\end{array}$ & $\begin{array}{c}36,0 \\
(33,7-38,3)\end{array}$ & $-0,3^{*}$ & $-3,6^{*}$ & $\begin{array}{c}59,4 \\
(57,3-61,5)\end{array}$ & $\begin{array}{c}70,0 \\
(68,1-71,8)\end{array}$ & $\begin{array}{c}67,2 \\
(64,9-69,4)\end{array}$ & $-10,6^{*}$ & $-7,8^{*}$ \\
\hline
\end{tabular}

Cap. $=$ capitais IMC $=$ índice de massa corporal; p.p. = pontos percentuais; ${ }^{*} \mathrm{p}<0,05$.

com a PNS Capitais $(\mathrm{CCC}=0,276)$ (Figura $2 \mathrm{~A}$ e B). Por outro lado, quando observamos as estimativas de inatividade no lazer (Figura $2 \mathrm{C}$ e D), é observada concordância baixa para ambas amostras da PNS ( $\mathrm{CCC}=0,056$ utilizando-se toda a amostra da PNS na comparação, e CCC = 0,202 utilizando a PNS Capitais), o que é percebido visualmente pela disposição dos pontos no gráfico, a qual revela que as estimativas da PNS foram consistentemente superiores àquelas obtidas pelo VIGITEL para todos os estados brasileiros.

\section{Discussão}

Os adultos considerados suficientemente ativos no lazer apresentaram frequências estatisticamente superior no VIGITEL quando comparados à PNS, sendo essas frequências mantidas quando o VIGITEL foi comparado à sub-amostra da PNS. Em relação ao transporte ativo, as estimativas entre os dois inquéritos foram mais comparáveis, todavia, apresentaram grande variabilidade, sendo as estimativas superestimadas ao comparar a sub-amostra de capitais da PNS com o VIGITEL. No que diz respeito ao tempo de TV o percentual foi superior na PNS capitais para todas as categorias de variáveis independentes, quando analisando todas as capitais conjuntamente. A PNS gerou estimativas superiores às do VIGTEL quando comparada a inatividade física no lazer. Ainda, estimativas de prevalência de indivíduos suficientemente ativos no lazer apresentaram concordância baixa, em relação às prevalências de realização de deslocamento ativo para o trabalho pode-se observar concordância moderada a alta e, levando em consideração o tempo de TV observou-se concordância moderada quando o VIGITEL foi comparado a amostra total da PNS e, baixa concordância quando comparado a PNS capitais. Baixa concordância para ambas as amostras da PNS foram observadas em 

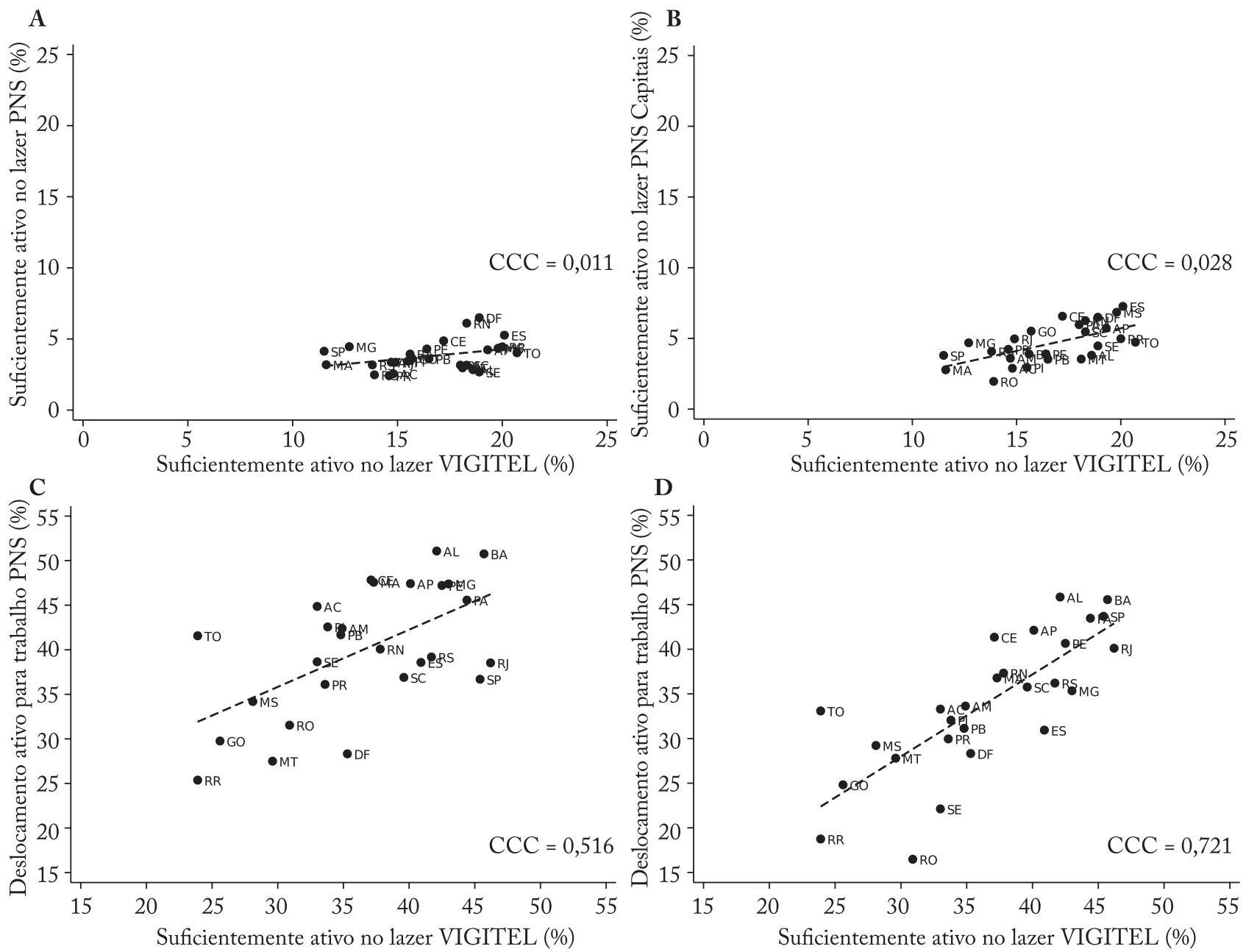

Figura 1 - Proporção de indivíduos em cada estado brasileiro suficientemente ativos no lazer comparando (A) PNS com VIGITEL e (B) PNS Capitais com VIGITEL, e proporção de indivíduos que se deslocam ativamente para o trabalho comparando (C) PNS com VIGITEL e (D) PNS Capitais com VIGITEL, durante o ano de 2013.

comparação ao VIGITEL.

Embora os inquéritos em saúde envolvendo a população brasileira tenham começado na década de 1990 com a condução da Pesquisa Nacional por Amostra de Domicílios (PNAD)22, perguntas envolvendo mais domínios do estilo de vida começaram a ser abordadas somente em 2009, pela Pesquisa Nacional de Saúde do Escolar (PeNSE) ${ }^{23}$. No mesmo ano, a VIGITEL ${ }^{24}$ também investigou o estilo de vida de adultos das capitais dos estados brasileiros. No entanto, somente em 2013 é que foi conduzida a PNS ${ }^{25}$ com o objetivo de monitorar o estilo de vida e as DCNTs.

Mesmo que estes inquéritos estejam interessados em assuntos similares, eles mostram-se diferentes quanto alguns fatores chave, como por exemplo a população alvo e os custos envolvidos. Os custos de um inquérito como a PNS, a qual é uma pesquisa de abrangência nacional com entrevistas domiciliares face-a-face, são bastante superiores aos do VIGITEL, que avalia por inquérito telefônico apenas moradores das capitais ${ }^{26}$. Mesmo assim, caso as prevalências das estimativas fossem semelhantes entre os inquéritos, seria possível a adoção do inquérito com menor custo e tempo de execução para o monitoramento dos desfechos estudados a fim da obtenção de estimativas confiáveis nacionalmente.

Estudo anterior, o qual utilizou dados somente das capitais, já havia encontrado diferenças nas estimativas entre a PNS e o VIGITEL, principalmente para a prática de atividade física no lazer ${ }^{12}$. Entretanto, a magnitude de diferença apresentada no presente estudo foi ainda maior, podendo ser atribuída ao modo de aplicação da pergunta sobre a prática de atividade física no lazer. $\mathrm{Na}$ PNS o entrevistado poderia relatar abertamente quantos dias por semana e quantas horas por dia ele praticava atividade física. No VIGITEL, essas questões tinham opções de resposta fechadas, por exemplo 1 a 2 dias, 3 a 

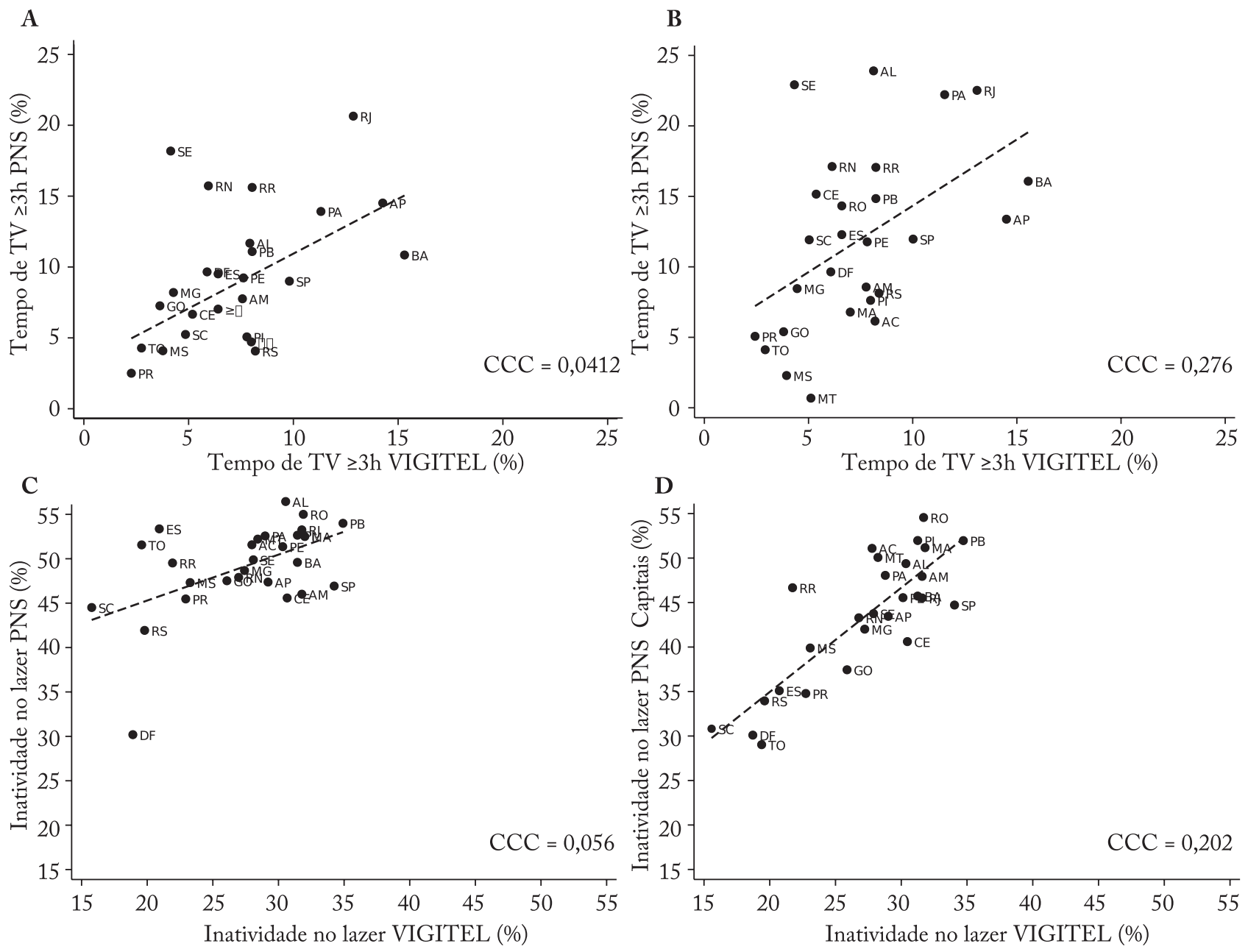

Figura 2 - Proporção de indivíduos em cada estado brasileiro com tempo de TV maior ou igual a três horas comparando (A) PNS com VIGITEL e (B) PNS Capitais com VIGITEL, e proporção inatividade no lazer comparando (C) PNS com VIGITEL e (D) PNS Capitais com VIGITEL, durante o ano de 2013.

4 dias, e 10 a 19 minutos, 20 a 29 minutos, etc. Esta falta de detalhamento da resposta no VIGITEL pode ter superestimado a prática suficiente de atividade física no lazer. Nesse sentido, incompatibilidades encontradas no presente estudo podem ser fruto dos diferentes métodos e questionários adotados no VIGITEL e PNS.

Com relação ao deslocamento ativo para o trabalho, foi encontrada concordância alta em virtude de ter sido aplicada exatamente a mesma pergunta em cada um dos inquéritos. Apesar disso, era esperado que o deslocamento ativo fosse mais prevalente na PNS do que no VIGITEL, visto que o deslocamento ativo é mais difundido entre indivíduos de menor nível socioeconômico, em maior concentração no interior dos estados ${ }^{27}$. Isso pode estar relacionado à limitação das linhas de telefone fixo entre pessoas com menor renda, mesmo que essa limitação seja levada em consideração para o cálculo do peso de amostragem ${ }^{13}$. Ainda, dado que as estimativas da PNS analisando somente capitais também apresentaram menor prevalência de deslocamento ativo para o trabalho ${ }^{17}$. O tempo sedentário, medido pelo tempo de TV maior ou igual a três horas por dia, foi um desfecho para o qual foi encontrada concordância moderada entre as estimativas dos inquéritos. $\mathrm{O}$ hábito de assistir televisão pode realmente ser similar entre capitais e municípios do interior. Apesar disso, caso fosse considerado o tempo de tela a partir do uso de computador e videogame, por exemplo, seria esperado que as estimativas do VIGITEL fossem superiores às da PNS, pois os indivíduos residentes em capitais teriam maior acesso a tais tecnologias.

As maiores diferenças entre as estimativas foram com relação à inatividade no lazer, onde as estimativas da PNS foram muito superiores àquelas obtidas pelo VIGITEL, mesmo que as perguntas aplicadas tenham sido iguais. Os dados analisados sugerem que a prática 
de atividade física no lazer é mais comum nas capitais do que nas cidades do interior dos país. No entanto, quando analisadas somente as capitais da PNS, a diferença quanto à inatividade física foi ainda maior, indicando dessa forma um possível viés em entrevistas telefônicas, corroborando com estudos anteriores que investigaram a comparação entre os inquéritos ${ }^{17}$.

O presente estudo encontrou que as estimativas referentes à prática de atividade física e tempo sedentário obtidas na PNS e no VIGITEL possuem moderada à baixa concordância. Dessa forma, reitera-se que ambos inquéritos possuem virtudes e limitações. Considera-se que os dados coletados no VIGITEL são de grande valia para a identificação de tendências e monitoramento de saúde ${ }^{28,29}$, bem como representativos das capitais brasileiras, tratando-se de alternativa viável em termos de custo e logística para acompanhar a população das capitais brasileiras. Por outro lado, a PNS é um inquérito para a vigilância do Brasil como um todo, o que possibilita a demarcação de áreas estratégicas para possíveis intervenções, incluindo regiões interioranas, bem como a estimativa de prevalências extrapoláveis para todo o território nacional.

Algumas limitações relacionadas aos estudos precisam ser pontuadas. A primeira é que não há um "padrão ouro" utilizado nacionalmente para comparação. Dessa forma, apenas inferências sobre as diferenças de respostas obtidas pelos inquéritos, ressalvando-se as discrepâncias metodológicas, puderam ser observadas. Ainda, aspectos peculiares de cada estudo específico devem ser considerados. Relacionado ao VIGITEL, consideramos a possibilidade de um viés de ordem socioeconômica, mesmo ajustando pelo peso de amostragem, devido ao fato de algumas capitais apresentarem baixa cobertura de telefonia fixa, o que também é modificado a cada ano ${ }^{17}$. Outro fator a ser considerado é a limitada compatibilidade entre as questões aplicadas pelos inquéritos. Embora ambos apliquem cerca de 20 questões sobre a prática de atividade física e comportamento sedentário, apenas cinco questões eram similares entre os dois inquéritos e foram utilizadas nas análises, sendo que as perguntas sobre tempo de televisão, deslocamento ativo e a inatividade no lazer eram idênticas, enquanto que a pergunta relacionada à prática suficiente de atividade física no lazer apresentava uma pequena diferença quando consideramos os dois inquéritos, o que poderia explicar parte da discordância encontrada ${ }^{15,18}$. Além disso, como as pesquisas ocorreram em épocas diferentes do ano, é plausível existir sazonalidade nos comportamentos.
Outra limitação está relacionada ao indicador adotado de IMC, o qual foi estimado a partir de variáveis auto relatadas que possuem um elevado número de perdas na presente amostra (cerca de 30\%). Nesse sentido, as características das perdas podem ser diferentes comparadas aos indivíduos incluídos nas análises. Análises comparando variáveis sócio-demográficas dos indivíduos com e sem informação de IMC, revelaram maior proporção de sujeitos sem escolaridade, sendo que indivíduos com menor escolaridade apresentam menores níveis de atividade física no lazer ${ }^{27}$. Com isso, acredita-se que as estimativas de indivíduos suficientemente ativos no lazer possam estar superestimadas, enquanto as estimativas de inatividade no lazer podem estar subestimadas.

As análises realizadas mostraram que as estimativas obtidas para os inquéritos PNS e VIGITEL são de concordância baixa à moderada para as questões relacionadas à prática de atividade física e comportamento sedentário, inclusive analisando somente as capitais da PNS. Apesar disso, os resultados não diminuem a importância da realização de qualquer um dos inquéritos, pois cada um abrange pontos importantes e complementares (em termos temporais, por exemplo) para o monitoramento e a vigilância de fatores relacionados à saúde da população brasileira. Os resultados aqui apresentados sugerem que a devida atenção deva ser tomada em relação às diferenças entre as estimativas geradas pelos inquéritos, de forma que as conclusões dos estudos e direcionamentos em políticas públicas considerem suas diferenças. Reforçamos a necessidade de que os instrumentos utilizados pelos diferentes inquéritos nacionais em saúde sejam padronizados, além de serem amplamente discutidos com a comunidade científica, visando obter um consenso.

\section{Conflito de interesses}

Os autores declaram não haver conflito de interesses.

\section{Contribuição dos autores}

Mendes GF, Häfele V, Blumenberg C, Werneck AO e Radicchi $\mathrm{M}$, participaram da concepção, análise e interpretação dos dados e redação do manuscrito, bem como da elaboração da primeira versão do manuscrito. Bielemann $\mathrm{R}$ e Coll C, revisaram o manuscrito e contribuíram substancialmente. Todos os autores aprovaram a versão final do manuscrito e declaram ser responsáveis por todos os aspectos do trabalho, garantindo sua precisão e integridade.

\section{Agradecimentos}

Os autores agradecem a CAPES, bem como a Fundação de Amparo à Pesquisa do Estado de São Paulo - FAPESP, pela bolsa 
de mestrado do autor AOW (processo 2017/27234-2) e Fundação de Amparo à Pesquisa do Estado do Rio Grande do Sul (FAPERGS), pela bolsa de mestrado do autor VH.

\section{Referências}

1. Lee IM, Shiroma EJ,Lobelo F,Puska P, Blair SN, Katzmarzyk PT, et al. Effect of physical inactivity on major noncommunicable diseases worldwide: An analysis of burden of disease and life expectancy. Lancet. 2012;380(9838):219-29.

2. Mielke GI, Hallal PC, Rodrigues GBA, Szwarcwald CL, Santos FV, Malta DC. Prática de atividade física e hábito de assistir à televisão entre adultos no Brasil: Pesquisa Nacional de Saúde 2013. Epidemiol Serv Saude. 2015;24(2):277-86.

3. Tremblay MS, Aubert S, Barnes JD, Saunders TJ, Carson $\mathrm{V}$, Latimer-Cheung AE, et al. Sedentary behavior research network (SBRN) - Terminology consensus project process and outcome. Int J Behav Nutr Phys Act. 2017;14:75.

4. Matthews CE, Keadle SK, Troiano RP, Kahle L, Koster A, Brychta R, et al. Accelerometer-measured dose-response for physical activity, sedentary time, and mortality in US adults. Am J Clin Nutr. 2016:1-9.

5. Ekelund U, Steene-Johannessen J, Brown WJ, Fagerland MW, Owen N, Powell KE, et al. Does physical activity attenuate, or even eliminate, the detrimental association of sitting time with mortality? A harmonized meta-analysis of data from more than 1 million men and women. Lancet. 2016;388(10051):1302-10.

6. Rezende LFM, Sá TH, Mielke GI, Viscondi JYK, ReyLópez JP, Garcia LMT. All-cause mortality attributable to sitting time: analysis of 54 countries worldwide. Am J Prev Med. 2016;51(2):253-63.

7. Atkin AJ, Gorely T, Clemes SA, Yates T, Edwardson C, Brage $\mathrm{S}$, et al. Methods of measurement in epidemiology: Sedentary behaviour. Int J Epidemiol. 2012;41:1460-71.

8. Keadle SK, Moore SC, Sampson JN, Xiao Q, Albanes D, Matthews CE. Causes of Death Associated With Prolonged TV Viewing. Am J Prev Med. 2015;49(6):811-21.

9. van de Ploeg HP, Chey T, Korda RJ, Banks E, Bauman A. Sitting time and all-cause mortality risk in 222497 Australian adults. Arch Intern Med. 2012;172(6):494-500.

10. Barros MBA. Inquéritos domiciliares de saúde:potencialidades e desafios. Rev Bras Epidemiol. 2008;11(1):6-19.

11. Francisco PMSB, Barros MBDA, Segri NJ, Alves MCGP. Comparison of estimates of population-based surveys. Rev Saude Publica. 2013;47(1):60-8.

12. Malta DC, Iser BPM, Santos MAS, Andrade SSA, Stopa SR, Bernal RTI, et al. Estilos de vida nas capitais brasileiras segundo a Pesquisa Nacional de Saúde e o Sistema de Vigilância de Fatores de Risco e Proteção para Doenças Crônicas Não Transmissíveis por Inquérito Telefônico (Vigitel), 2013. Rev Bras Epidemiol. 2015;18:68-82.

13. Ministério da Saúde. Plano de ações estratégicas para o enfrentamento das doenças crônicas não transmissíveis (DCNT) no brasil 2011-2022.; 2011. [citado em 2019 mai 10]. Disponível em: http://bvsms.saude.gov.br/bvs/ publicacoes/plano_acoes_enfrent_dcnt_2011.pdf.

14. IBGE. Pesquisa Nacional de Saúde 2013. 2014. 181 p. [citado em 2017 jan 20]. Disponível em: htp://ftp.ibge.gov. br/PNS/2013/pns2013.pdf.
15. Ministério da Saúde (BR). Secretaria de Vigilância em Saúde. Secretaria de Gestão Estratégica e Participativa. Vigitel Brasil 2013: vigilância de fatores de risco e proteção para doenças crônicas por inquérito telefônico [Internet]. Brasília: Ministério da Saúde, 2013. [citado em 2019 mai 10]. Disponível em: http://bvsms.saude.gov.br/bvs/publicacoes/ vigitel_brasil_2013.pdf.

16. Groves RM. Three eras of survey research. Public Opin Q. 2011;75(5 SPEC. ISSUE):861-71.

17. Bernal RT, Malta DC, Morais Neto OL, Claro RM, Mendonça BCA, Oliveira ACC, et al. Vigitel - Aracaju, Sergipe, 2008: efeitos da pós-estratificação na correção de vícios decorrentes da baixa cobertura de domicílios com telefone fixo. Rev Bras Epidemiol. 2014;17:163-74.

18. Malta DC, Andrade SSCA, Stopa SR, Pereira CA, Szwarcwald CL, Júnior S, et al. Brazilian lifestyles: National Health Survey results, 2013. Epidemiol Serv Saude. 2015;24(2):217-26.

19. Souza-Júnior PRB, Freitas MPS, Antonaci GA, Szwarcwald CL. Desenho da amostra da Pesquisa Nacional de Saúde 2013. Epidemiol e Serviços Saúde. 2015;24(2):207-16.

20. World Health Organization - W.H.O. Obesity: Preventing and Managing the Global Epidemic. 2000:6-8.

21. Lin LI. A concordance correlation coefficient to evaluate reproducibility. Biometrics. 1989;45(1):255-68.

22. Lima-Costa MF, Matos DL, Camargos VP, Macinko J. Tendências em dez anos das condições de saúde de idosos brasileiros: evidências da Pesquisa Nacional por Amostra de Domicílios (1998, 2003, 2008). Cien Saude Colet. 2011;16(9):3689-96.

23. Instituto Brasileiro de Geografia e Estatística - IBGE. Censo Demográfico 2010. [citado em: 2016 out]. Disponível em: http://www.censo2010.ibge.gov.br.

24. Ministério da Saúde (BR). Secretaria de Vigilância em Saúde. Secretaria de Gestão Estratégica e Participativa. Vigitel Brasil 2009: vigilância de fatores de risco e proteção para doenças crônicas por inquérito telefônico [Internet]. Brasília: Ministério da Saúde, 2010.

25. Szwarcwald CL, Malta DC, Pereira CA, et al. Pesquisa Nacional de Saúde no Brasil: concepção e metodologia de aplicação. Cien Saude Colet. 2014;19(2):333-42.

26. Moura EC, Morais Neto OL, Malta DC, Moura L, Silva NN, Bernal R, et al. Vigilância de Fatores de Risco para Doenças Crônicas por Inquérito Telefônico nas capitais dos 26 estados brasileiros e no Distrito Federal (2006). Rev Bras Epidemiol. 2008;11:20-37.

27. Silva SG, Del Duca GF, Silva KS, Oliveira ESA, Nahas MV. Commuting to and from work and factors associated among industrial workers from Southern Brazil. Rev Saude Publica. 2012;46(1):180-4

28. Hallal PC, Knuth AG, Reis RS, Rombaldi AJ, Malta DC, Iser BPM, et al. Time trends of physical activity in Brazil (2006-2009). Rev Bras Epidemiol. 2011;14 Suppl1:53-60.

29. Mielke GI, Hallal PC, Malta DC, Lee IM. Time trends of physical activity and television viewing time in Brazil: 20062012. Int J Behav Nutr Phys Act. 2014;11:101.

Recebido: 04/02/2019

Aprovado: 25/05/2019

Como citar este artigo:

Mendes GFF, Häfele V, Blumenberg C, Werneck AO, Radicchi MR, Coll CVN, et al. Comparação das estimativas de atividade física e comportamento sedentário em adultos brasileiros no VIGITEL e PNS, Brasil, 2013. Rev Bras Ativ Fís Saúde. 2018;23:e0062.

DOI: $10.12820 /$ rbafs.23e0062 